\title{
Optimización del Algoritmo Genético para la Solución Integral de Enrutamiento en Redes Fotónicas
}

Arturo B. Rodríguez ${ }^{1}$ y Fideromo Saavedra ${ }^{2}$

(1) Universidad del Pacífico, Escuela de Ingeniería Informática, Av. Las Condes 11121, Las Condes, Santiago-Chile (e-mail: arodriguez@upacifico.cl)

(2) Universidad de Santiago de Chile, Facultad de Ingeniería, Departamento Ing. Eléctrica, Av. Alameda Libertador Bernardo O'Higgins 3363, Estación Central, Santiago-Chile (email: fideromo.saavedra@usach.cl)

Recibido Jun. 01, 2009; Aceptado Ago. 05, 2009; Versión Final recibida Ago.31, 2009

\begin{abstract}
Resumen
Este artículo busca una nueva forma de solucionar el problema de enrutamiento y asignación de longitud de onda en redes fotónicas (RWA - Routing Wavelength Assignement). Este problema ha sido resuelto dividiéndolo en 2 subproblemas, y aplicando diferentes algoritmos optimizadores, entre ellos se encuentran los algoritmos genéticos, el mismo que se utiliza en esta investigación, sin embargo se ha propuesto la solución sin dividirlo, resolviéndolo de manera integral. La investigación analiza algunos parámetros de los algoritmos genéticos, y se optimizan para la posterior utilización en la resolución del problema RWA. Para ello, se realizaron simulaciones en escenarios de tráfico dinámico en la red NSFNET. Se logró determinar la posición de la reproducción, el tamaño de reproducción y la mortandad, que optimizan el algoritmo, en este trabajo no se utilizó mutación, debido a su poca relevancia en mejorar el algoritmo.
\end{abstract}

Palabras clave: red, NSFNET, algoritmos genéticos, longitud de onda, RWA.

\section{Optimization of Genetic Algorithms for the Integral Solution of Routing Wavelength Assignement}

\begin{abstract}
In presented article, it looks for a new form to solve to the problem of routing and allocation of wavelength in networks photons (RWA - Routing Wavelength Assignement). This problem has been solved dividing it in 2 subproblems, and applying to different algorithms optimizers, among them are the genetic algorithms, he himself who is used in this investigation, nevertheless has seted out the solution without dividing it, solving it of integral way. The investigation analyzes some parameters of the genetic algorithms, and they are optimized for the later use in the resolution of problem RWA. For it, simulations were made in scenes of dynamic traffic in network NSFNET. It was managed to determine the position of the reproduction, the size of reproduction and the loss of life, that optimizes the algorithm, in this work was not used mutation, due to its little relevance in improving the algorithm. Later simulations of the optimized algorithm were made in the same network, under dynamic traffic, these results will be shown in a next publication.
\end{abstract}

Keywords: network, NSFNET, genetic algorithms, wavelength, RWA 


\section{INTRODUCCIÓN}

Las tecnologías WDM logran aumentar la capacidad dada por la fibra óptica la cual es explotada con multiplexación por división de tiempo TDM (Chu y Li, 2003). Cada longitud de onda es guiada por la fibra a la que se denomina canal WDM. Los sistemas de comunicación multiplexados por longitud de onda han aumentado debido a la demanda de las redes de acceso por un transporte de mayor velocidad, y a la aparición de nuevos dispositivos ópticos, con integración de inteligencia, lo que permitiría en el mediano plazo la maduración de la arquitectura denominada IP sobre WDM (Schwartz, 2000; Xue y Zhang, 2006; Sahasrabuddhe y Ramamurthy, 2002). Por lo tanto, la necesidad de establecer un método de cálculo de rutas y de asignación de longitud de onda es un problema de relevante importancia. A este problema se le denomina RWA (Routing Wavelength Assignement - Enrutamiento y Asignación de Longitud de Onda), el cual es resuelto con diversos algoritmos que están orientados a minimizar o maximizar alguna condición relevante en la red óptica como puede ser el retardo en la ruta, la utilización de longitudes de onda, la congestión de la red, la probabilidad de bloqueo, etc. (Kuri et al., 2003).

El problema es resuelto, por lo general, subdividiendo el problema en 2 partes, la primera determina la ruta a seguir, minimizando alguna condición pre-establecida, para lo cual se utilizan métricas con señalización basada en el estado de enlace o vector distancia, dependiendo de la condición a optimizar. La segunda determina la longitud de onda a utilizar, lo que es importante en redes sin cambio de longitud de onda, pues se debe respetar la condición restrictiva de continuidad de la longitud de onda en todos los enlaces que conforman la ruta (Rodríguez et al., 2004). Debido a que simular y resolver el problema de manera integral, tiene un elevado costo computacional, se realizó una optimización de los parámetros del algoritmo para su posterior utilización en la solución. Este trabajo muestra los resultados de la optimización del algoritmo genético, y en una próxima publicación se mostrarán los resultados de la simulación en la red NSFNET.

\section{METODOLOGÍA}

\section{Descripción del problema RWA}

En las redes totalmente ópticas (AON) los transmisores están conectados con cada receptor en todas las longitudes de onda. Cuando muchas sesiones utilizan la misma longitud de onda, estas señales no se pueden enviar sobre la misma fibra simultáneamente, para evitar las colisiones dentro de la red y por consecuencia el bloqueo de la sesión. Se debe asegurar que si un receptor está en espera de datos, en una la longitud de onda determinada en un intervalo de tiempo determinado, solo una señal con esa longitud de onda debe llegar al receptor, si 2 o más señales llegan bajo esas condiciones estamos ante un fenómeno llamado CONTENCION (Ramaswami y Kumar, 1995).

Se definen algunas variables para la formulación del problema, se sabe que en la red existe un tráfico actual, y un tráfico por llegar o solicitado. Este tráfico puede ser estático o dinámico, estático cuando $\overline{T_{l l}}$, el tiempo entre llegadas de solicitudes a los nodos fronterizos (Edge Router) es menor a $\bar{t}_{c}$ que es el tiempo de tenencia de la conexión de las solicitudes (Holding Time), o dinámico en el caso contrario. El problema es establecer rutas y la longitud de onda en la red para condiciones de optimización como, mínima probabilidad de bloqueo, baja latencia de transporte en la ruta, bajo nivel de jitter, etc. El problema RWA-Routing Wavelength Assignment (enrutamiento y asignación de longitud de onda), en el escenario dinámico es conocido como NP-completo, es decir el tiempo de solución es desconocido debido a la variabilidad del espacio de solución (Saengudomiert et al., 2005). Es por esta razón que los esfuerzos han sido centrados en generar mejores algoritmos, y se han probado de muchas maneras; la mayoría de las veces dividiendo el problema. Las rutas ópticas están formadas por enlaces, los cuales direccionan el haz de luz (datos) a través de la misma longitud de onda, sin embargo esta posibilidad se agota cuando la demanda supera el número de sesiones posible bajo esta restricción; cuando no se puede satisfacer esta restricción, es cuando se bloquea la solicitud (Zang et al., 2000). 


\section{Red NSFNET}

La red utilizada para llevar acabo la simulación fue la NSFNET (Nacional Science Foundation Network), el NSFNET Backbone o sistema principal de conmutación de la National Science Foundation NETwork; como se puede observar en la figura 1.

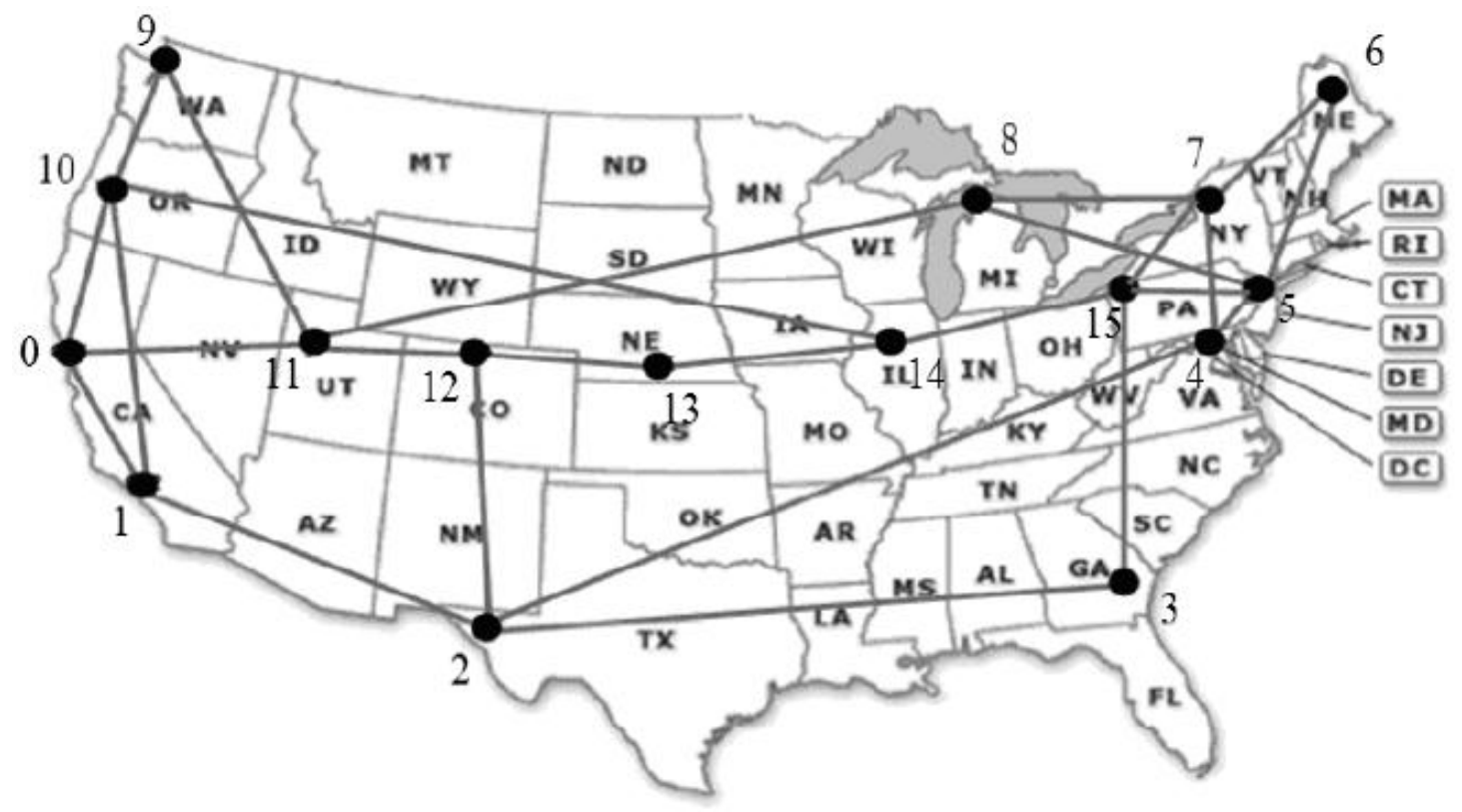

Fig. 1: Backbone NSFNET

La red de la figura 1, trabaja con tecnología WDM, lo cual permite utilizarla como referente para nuestra simulación. Muchos trabajos se han realizado tomando como base esta red, sin embargo pocos han utilizado métodos que solucionan el RWA de manera integral. El backbone cuenta con 16 nodos y 25 enlaces, esto determina que tiene una capacidad instalada del $20.8 \%$ de una capacidad de 120 enlaces como máximo.

\section{Algoritmo genético}

La ruta utilizada en la solución se asemeja a un cromosoma, la cual está representada por un conjunto de $N$ números, y cada número representa un conmutador óptico en la red fotónica, cada uno de estos números se define en adelante como gen, y el conjunto como cromosoma.

Sea $P_{0}$ la población inicial del algoritmo genético a utilizar, y $n_{P}$ el número de cromosomas que forman esta población inicial, además se ejecutarán $m$ (siendo $m<n_{P}$ ) algoritmos genéticos para los $m$ nodos que solicitan servicio de transporte, en la red de $N$ nodos. Dentro de los algoritmos genéticos existen diversas implementaciones que permiten diferentes comportamientos de $n_{p}$, para nuestro caso este valor se mantiene constante y es un parámetro de la simulación. De tal forma que, se define el arreglo tridimensional $P_{0}$ de $n_{P} \times\left(N+3 n_{W}\right) \times N$ elementos, siendo $n_{W}$ el número de longitudes de onda por enlace, considerado constante en cada enlace.

$P=\left\{\begin{array}{l}p_{a b c} / p_{a b c}=t \wedge a \in\left[0, n_{P}-1\right] \wedge b \in\left[0, N+3 n_{W}+2\right] \\ \wedge c \in[0, m-1] \wedge t \in[0, N-1]\end{array}\right\}$

Esta matriz es llenada de manera aleatoria, las columnas adicionales son para guardar los cálculos de la función aptitud referente a cada longitud de onda disponible. 
El primer y último gen deben ser el origen y destino de la solicitud, el resto de genes se rellenan aleatoriamente en la matriz poblacional, cada plano frontal (Matriz poblacional) que va en la dirección de los nodos emisores, es utilizada para calcular la ruta y longitud de onda de la solicitud instantánea de ese nodo, se expone el nodo $k+1$ separado para su mejor observación (nótese que la matriz empieza en 0). (Ver figura 2)

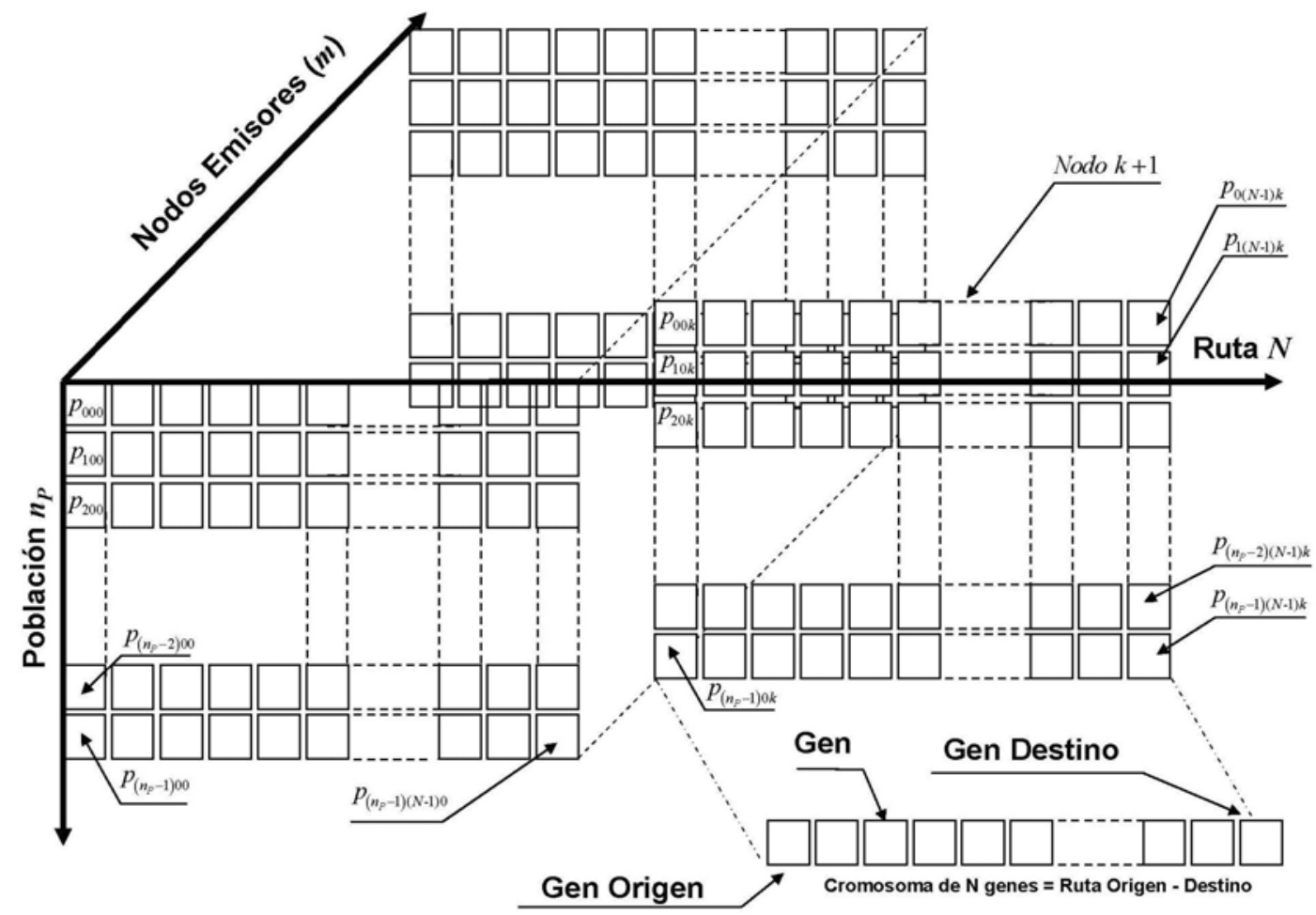

Fig. 2: Estructura matricial de la población inicial de los $m$ nodos emisores.

\section{Función Aptitud}

Debido a que en la mayoría de los casos el problema RWA ha sido tratado subdividiendo el problema en 2 subproblemas, nosotros evitaremos esta técnica, planteando una función aptitud que permita el cálculo de la ruta y detecte la longitud de onda disponible respetando la continuidad de longitud de onda para la ruta seleccionada.

La función de aptitud busca un resultado entero que permita encontrar la misma longitud de onda en cada uno de los enlaces utilizados en la ruta. Es necesario indicar que el algoritmo genético no busca una ruta óptima, solo busca una buena ruta. Se define:

$e_{\text {Max }}=\left\|\frac{V T_{1}}{V T_{4}}\right\|$

$V T_{1}=$ Velocidad de Transmisión de Capa 1, para este caso: $V T_{1}=2.5 \mathrm{Gbps}$.

$V T_{4}=$ Velocidad de Transporte, para este caso: $V T_{4}=155.52 \mathrm{Mbps} .=0.15552$ Gbps. (SDH-STM1/SONET )

$e_{\text {Max }}$, indicará el valor máximo de conexiones para cada longitud de onda en cada enlace, este valor deberá ir variando a medida que se satisfacen las solicitudes que llegan a los routers y se terminen los estados de conexión de las rutas de luz activas (lightpath).

$E M X=\left\{\begin{array}{l}e_{i j k} /\left(e_{i j k}=0 i=j \wedge e_{i j k}=G N E E \wedge e_{i j k}=e_{\text {Max }} S E E\right) \\ \forall i \in[0, N-1] \wedge j \in[0, N-1] \wedge k \in\left[0, n_{w}-1\right]\end{array}\right\}$

$N E E=$ No Existe Enlace. $\quad S E E=$ Si Existe Enlace, donde $N$ es el número de nodos. 
$C_{p q}^{k}$, como el producto del estado del enlace en la Matriz $E M X$, estado de la matriz $\lambda$ y estado de la matriz $T$, para el enlace $(p+1, q+1)$ y la $(k+1)$-ésima longitud de onda.

$C_{p q}^{k}=E M X_{p q} \lambda_{p q k} t_{p q k}$

$C_{p q}$, como el estado del enlace en la Matriz EMX, para el enlace. $(p+1, q+1)$

$C_{p q}=E M X_{p q}$

De tal forma que la función aptitud será, el cociente:

$F A_{(k)}=\frac{\sum_{r=0}^{N-2} \mathrm{C}_{\mathrm{r}(\mathrm{r}+1)}^{\mathrm{k}}}{\sum_{r=0}^{N-2} \mathrm{C}_{\mathrm{r}(\mathrm{r}+1)}}$

Cuando, FA tenga un resultado entero significará que los enlaces conformantes estarán utilizando la misma longitud de onda, además el ordenamiento secuencial ascendente del algoritmo, establecerá encontrar, después de un número de iteraciones, una ruta con condiciones de FA entero. Si $C_{p q}^{k}$ tiene un valor negativo, será porque la longitud de onda en el enlace está en uso.

\section{Reproducción}

El proceso reproductivo es considerado una variable de simulación, este proceso es un intercambio intergenético entre cromosomas denominados padres para obtener los cromosomas hijos. Sea:

Antes de la reproducción:

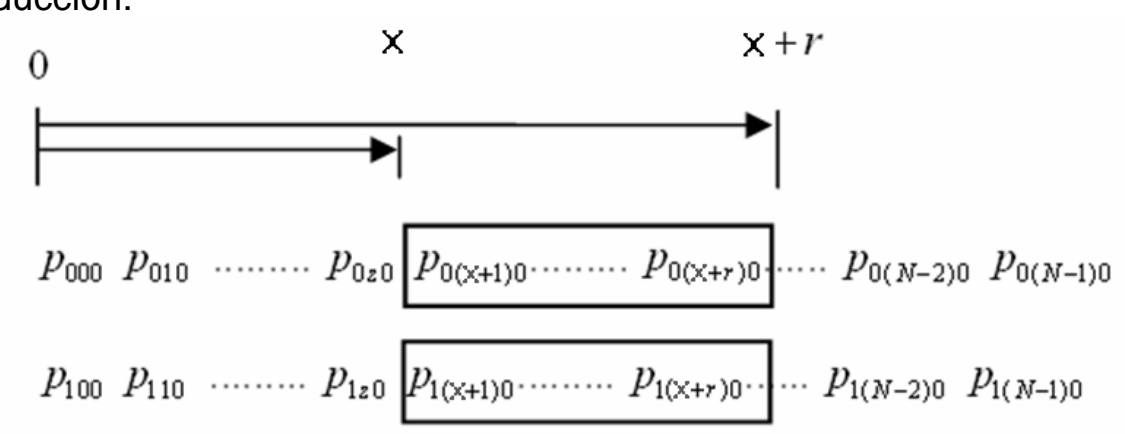

Después de la reproducción:

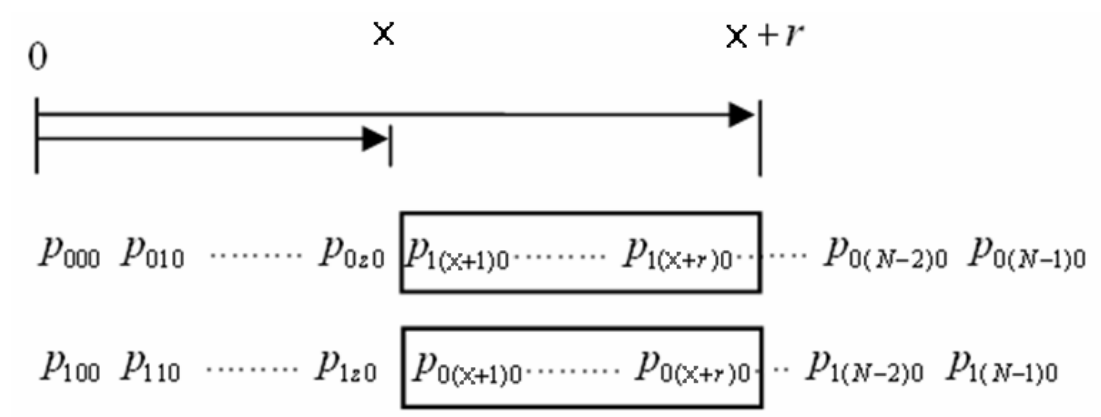


Dónde, $x$ es la posición donde empieza la reproducción del cromosoma y $r$ es el largo de la reproducción. Después de analizar las posibles combinaciones, podemos observar:

$x \in[1, N-4] \wedge r[1, N-x-3]$

Por ejemplo para N=16, y x=3 $r=7$ se tendrá: GGGRRRRRRRGGGGGG

Dónde G representa el Gen del cromosoma sin reproducir, y R representa el Gen del cromosoma reproducido. Bajo estas condiciones de variabilidad reproductiva; se tienen una cantidad de escenarios de simulación dados por la ecuación:

$$
N_{E}=\frac{N(N-3)}{2}
$$

Para este trabajo $\mathrm{N}=16$, por lo tanto se simularon $N_{E}=104$ escenarios reproductivos.

\section{Mutación}

Este proceso realiza el cambio aleatorio de uno o más genes, ubicados en diferentes posiciones dentro del cromosoma, el número de cromosomas podrían ser de 1 a N-1 (exceptuando el Gen mutado anteriormente), sin embargo, a medida que se aumenta la cantidad de genes mutados, esto va afectando negativamente a la convergencia del algoritmo. Este proceso no es siempre beneficioso, sin embargo muy a menudo se muta solo el primer Gen de la segunda mitad.

\section{Mortandad}

La etapa de mortandad del algoritmo genético, es la eliminación de los cromosomas menos aptos, esto está asociado a una política de mortandad, y se expresa como un porcentaje del total de la población $n_{p}$, la mortandad es una variable de simulación y se varió en el intervalo porcentual $[5,95]$, se realizaron simulaciones aumentando la mortandad cada $5 \%$.

Para el caso del criterio de parada del algoritmo, se propuso el número de iteraciones $n_{I}$, variando en la simulación desde 100 hasta 1000. Es decir:

$n_{I} \in[100,1000]$

El algoritmo una vez alcanzado el número de iteraciones máximo dado por $n_{I}$, se detiene y empieza a resolver las nuevas solicitudes de cada nodo.

\section{Actualización de Matrices $E M X, \lambda$ y $T$}

Cada vez que se encuentre una solución a la solicitud de servicios de los nodos, las matrices de longitudes de onda, de conexiones y de tiempos deben actualizarse, para poder determinar las nuevas rutas bajo el nuevo escenario.

En el caso de la matriz de longitud de ondas $\lambda$, la longitud de onda elegida en los enlaces de la ruta elegida deberán colocarse en 0 , para de esta manera el algoritmo no la utilice, este valor se mantendrá en 0 hasta que la matriz de tiempos indique la finalización de la conexión por cumplimiento del servicio de conexión solicitado. Una vez culminado este tiempo, la longitud de onda deberá actualizar a su valor por defecto; para poder ser utilizada nuevamente. 
En el caso de la matriz de conexiones $E M X$, esta deberá ser actualizada similarmente a la anterior, pero disminuyendo la cantidad de conexiones en los enlaces de acuerdo al número de conexiones solicitadas en el servicio, estas conexiones se recuperarán (aumentarán), una vez culminado el estado de conexión. En el caso de la matriz de tiempos $T$, esta matriz indicará el tiempo de conexión en cada enlace por cada longitud de onda (Matriz tridimensional idéntica a la matriz $\lambda$ ), un thread se encargará de disminuir los tiempos a medida que el tiempo transcurra. Una vez que se termine el tiempo de conexión, las matrices $\lambda$ y EMX serán actualizadas.

\section{RESULTADOS Y DISCUSIÓN}

La simulación se llevó a cabo manteniendo una demanda estática es decir una carga constante de 300 Erlang, con la finalidad de trabajar bajo stress. La simulación se codificó en una plataforma de programación $\mathrm{C}++$, por su versatilidad con lo threads, además se utilizaron 8 lambdas en los enlaces, se hizo la suposición que cada enlace tenía la capacidad de trasmitir y recepcionar 8 lambdas. Las variables de simulación fueron, la mortandad, el número de iteraciones, población, la probabilidad de emisión, así como la posición de la ventana de reproducción que viene dada por el par $(x, r)$, donde $x$ la posición de la ventana y $r$ indica la longitud de la ventana. De tal forma que en la tabla 1 , se muestra la probabilidad de bloqueo, frente a cada una de las variables, también se midió la utilización de la red en cada escenario de simulación sin embargo no se muestran en este trabajo.

Tabla 1: Resultados de la probabilidad de bloqueo, comparada con las ventanas de reproducción y su posición.

\begin{tabular}{|c|c|c|c|c|c|c|c|c|c|c|c|c|c|c|}
\hline$z v$ & $\mathbf{1}$ & $\mathbf{2}$ & $\mathbf{3}$ & $\mathbf{4}$ & $\mathbf{5}$ & $\mathbf{6}$ & $\mathbf{7}$ & $\mathbf{8}$ & $\mathbf{9}$ & $\mathbf{1 0}$ & $\mathbf{1 1}$ & $\mathbf{1 2}$ & $\mathbf{1 3}$ & PMDO \\
\hline $\mathbf{1}$ & 0.63 & 0.44 & 0.56 & 0.55 & 0.16 & 0.31 & 0.28 & 0.08 & 0.26 & 0.49 & 0.36 & 0.35 & 0.55 & 0.39 \\
\hline $\mathbf{2}$ & 0.81 & 0.82 & 0.38 & 0.49 & 0.27 & 0.22 & 0.34 & 0.48 & 0.50 & 0.52 & 0.50 & 0.59 & 0.46 & 0.49 \\
\hline $\mathbf{3}$ & 0.80 & 0.64 & 0.45 & 0.59 & 0.47 & 0.32 & 0.29 & 0.20 & 0.49 & 0.42 & 0.46 & 0.54 & & 0.47 \\
\hline $\mathbf{4}$ & 0.91 & 0.92 & 0.67 & 0.47 & 0.31 & 0.25 & 0.39 & 0.36 & 0.40 & 0.26 & 0.41 & & & 0.49 \\
\hline $\mathbf{5}$ & 0.90 & 0.75 & 0.60 & 0.49 & 0.31 & 0.30 & 0.31 & 0.20 & 0.39 & 0.37 & & & & 0.46 \\
\hline $\mathbf{6}$ & 0.84 & 0.79 & 0.53 & 0.44 & 0.32 & 0.25 & 0.26 & 0.25 & 0.24 & & & & & 0.44 \\
\hline $\mathbf{7}$ & 0.96 & 0.55 & 0.54 & 0.60 & 0.43 & 0.46 & 0.44 & 0.41 & & & & & & 0.55 \\
\hline $\mathbf{8}$ & 0.88 & 0.72 & 0.65 & 0.58 & 0.37 & 0.37 & 0.18 & & & & & & & 0.54 \\
\hline $\mathbf{9}$ & 0.88 & 0.76 & 0.52 & 0.39 & 0.51 & 0.17 & & & & & & & & 0.54 \\
\hline $\mathbf{1 0}$ & 0.79 & 0.57 & 0.48 & 0.45 & 0.52 & & & & & & & & & 0.56 \\
\hline $\mathbf{1 1}$ & 0.97 & 0.82 & 0.59 & 0.40 & & & & & & & & & & 0.70 \\
\hline $\mathbf{1 2}$ & 0.67 & 0.54 & 0.78 & & & & & & & & & & & 0.66 \\
\hline $\mathbf{1 3}$ & 0.77 & 0.82 & & & & & & & & & & & & 0.80 \\
\hline $\mathbf{1 4}$ & 0.65 & & & & & & & & & & & & & 0.65 \\
\hline PMDO & 0.82 & 0.70 & 0.56 & 0.50 & 0.37 & 0.87 & 0.31 & 0.28 & 0.38 & 0.41 & 0.43 & 0.49 & 0.51 & \\
\hline
\end{tabular}

En la figura 3, se puede observar el comportamiento de la probabilidad de bloqueo y su variación frente a el tamaño $\mathrm{R}$ de la ventana de reproducción, se puede notar que cuando la ventana es menor a la mitad, la probabilidad de bloqueo es alta, y cuando la ventana pasa la mitad empieza a aumentar, es claro que el tamaño de óptimo de la ventana de reproducción es cercana a la mitad de la cantidad de nodos que conforman la red óptica.

La figura 4, muestra el comportamiento de la probabilidad de bloqueo promedio para cada ventana de reproducción medida, se puede observar que la mínima probabilidad ocurre cerca de la mitad de la cantidad de nodos en la red.

En la figura 5, se muestra el comportamiento de la probabilidad de bloqueo mientras varia la posición de la ventana de reproducción, y se observa que desde el inicio y hasta cerca la mitad del cromosoma la tendencia de la probabilidad es mínima. 


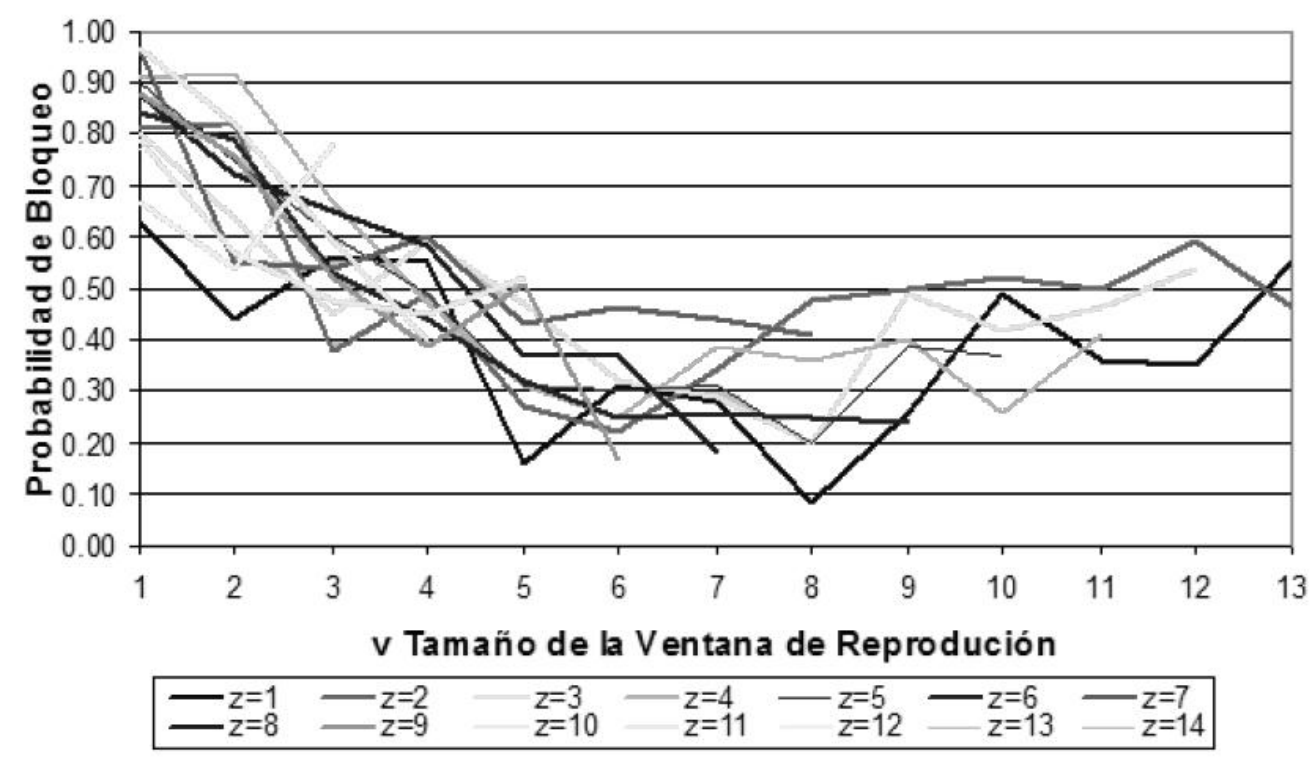

Fig. 3: Probabilidad de bloqueo de las diferentes ventanas de reproducción.

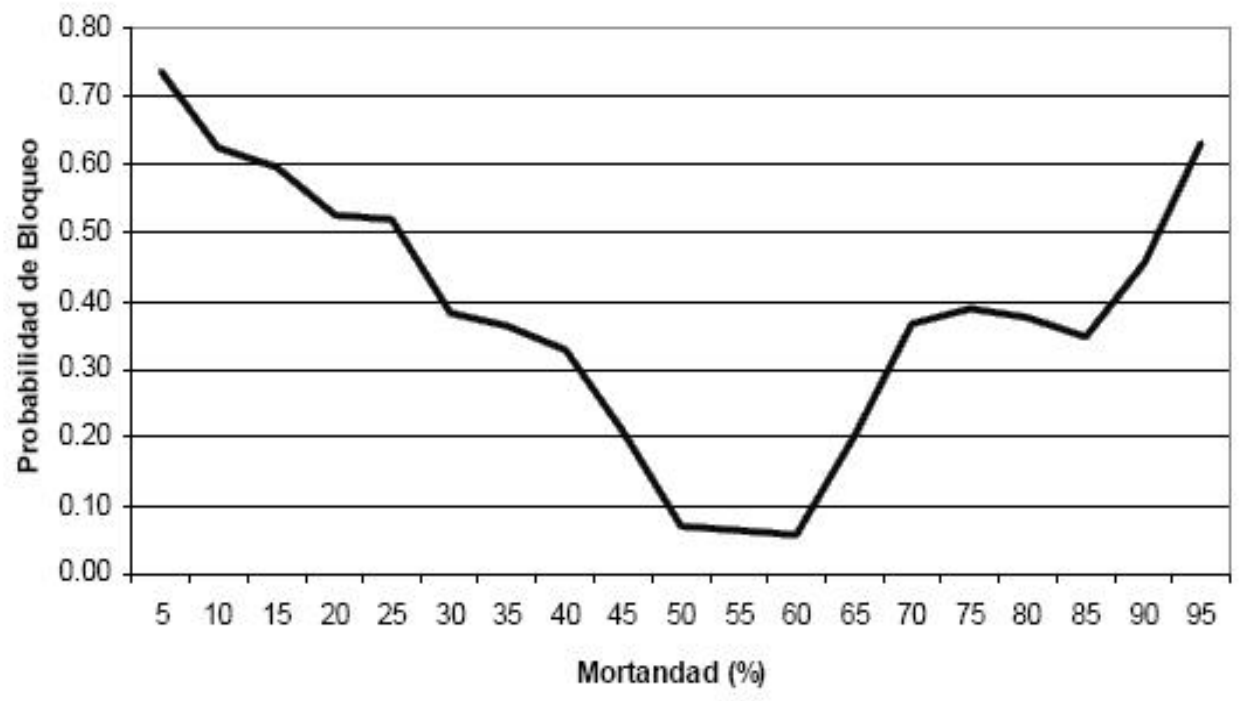

Fig. 4: Probabilidad de bloqueo promedio en las diferentes ventanas de reproducción.

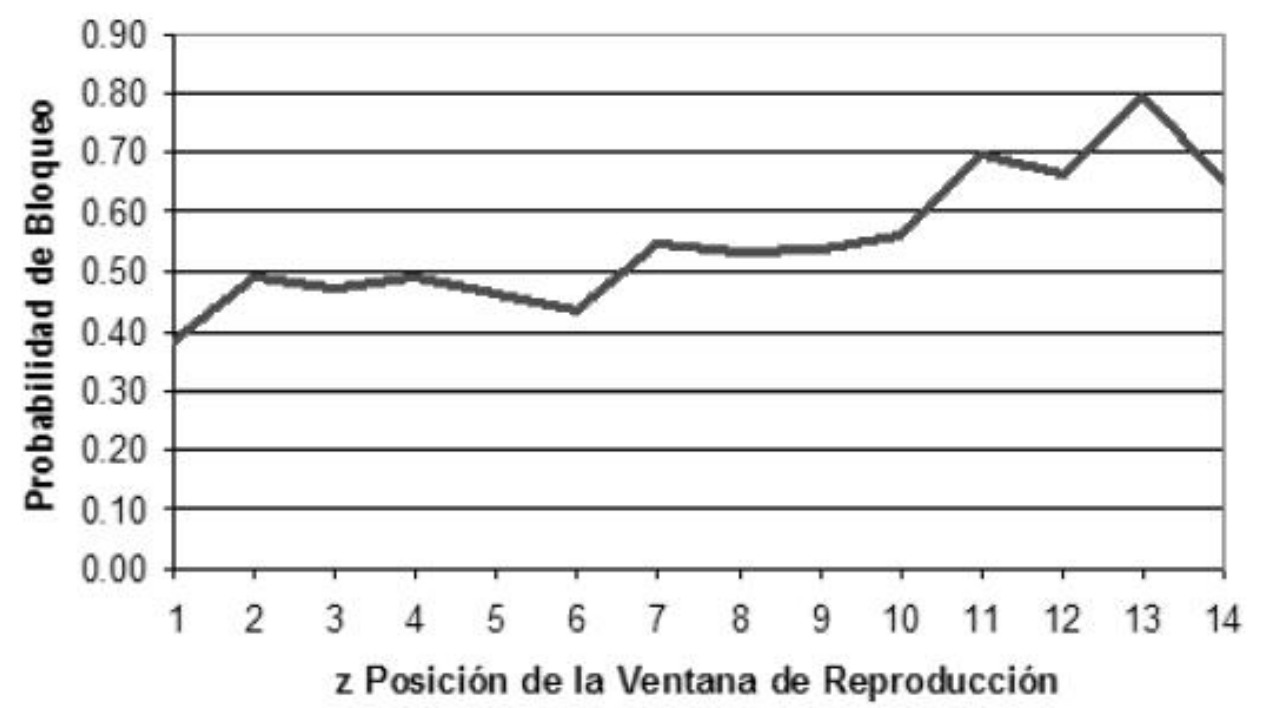

Fig. 5: Probabilidad de Bloqueo en las diferentes posiciones de la ventana de reproducción. 


\section{CONCLUSIONES}

La optimización de los algoritmos genéticos con una estrategia integradora (solucionar el problema simultáneamente), permitirá simplificar la solución, evitando la utilización de algoritmos de asignación de longitud de onda, sin embargo debemos aceptar el aumento de la complejidad de la solución. Durante la simulación, se observó que las limitaciones de la simulación vienen dadas por la falta de memoria y recursos de procesamiento a medida que aumentan los nodos de la red y longitudes de onda en la múltiplexación, debido a que las matrices utilizadas complejizan el procesamiento. Además la baja interconectividad (cantidad de enlaces) de los nodos, el cual es cerca del $20 \%$ hace que la solución se retarde más (Zang et al., 2000). Los resultados obtenidos permiten determinar que mientras mayor es la población utilizada en el algoritmo genético menor es la probabilidad de bloqueo, sin embargo la latencia de procesamiento aumenta, lo cual no es beneficioso. El efecto de la posición de la ventana de reproducción es relevante desde el comienzo hasta cerca de la mitad del cromosoma, debido a que la probabilidad de bloqueo es de tendencia menor. Así mismo el tamaño de la ventana de reproducción genera una disminución de la probabilidad de bloqueo a medida que aumenta hasta un tamaño cerca de la mitad del cromosoma. Además la mortandad es un aporte a la optimización en la medida que se acerque al $60 \%$ de la población de cromosomas. Por lo tanto, se lograron determinar los parámetros que optimizan el algoritmo y se mostrarán los resultados comparativos en una próxima publicación.

\section{REFERENCIAS}

Chu X., y Bo Li; A Dynamic RWA Algorithm in a Wavelength-Routed All-Optical Network with Wavelength Converters, INFOCOM 2003. Twenty-Second Annual Joint Conference of the IEEE Computer and Communications Societies. IEEE: 3, 1795-1804 (2003).

Kuri J., N. Puech y M., Gagnaire; Routing and Wavelength Assignement of Scheduled Lightpath Demands, IEEE Journal On Selected Areas in Communications: 21(8), 1231-1240 (2003).

Ramaswami R. y N. Kumar; Routing and wavelength assignment in all-optical networks, IEEE/ACM Transactions on Networking (TON): 3(5), 489-500 (1995).

Rodríguez A., F. Saavedra y M. Arias; Aplicación de algoritmos genéticos en la configuración óptima de enrutadores, Congreso Internacional de Telecomunicaciones SENACITEL, Valdivia, Chile 24 al 27 de Noviembre (2004).

Saengudomlert P., E.H. Modiano y R.G. Gallager; Dynamic Wavelength Assignment for WDM AllOptic Tree Networks, IEEE/ACM Transactions on Networking (TON): 13 (4), 895-905 (2005).

Sahasrabuddhe L. y S. Ramamurthy, Fault Management in IP Over WDM Networks: WDM Protection Versus IP Restoration, IEEE Journal on selected area in communications, 20 (1), 21-33 (2002).

Schwartz, M.; Computer-Communication Network Design and Analysis, Prentice-Hall, Inc., Amy Copley, Optical domain service interconnect: Defining mechanisms for enabling on-demand highspeed capacity from the optical domain, IEEE Communications: 38 (10), 165-174 (2000).

Xue, L., y Q. Zhang; Investigation of Uniform Models for Analysing Network Blocking Probability in ASON, Key Laboratory of Optical Communication \& Lightwave Technologies, Ministry of Education, Beijing University of Posts and Telecommunications: 1(1), 1-4 (2006).

Zang, H., J.P. Jue y B. Mukherjee; A Review of Routing and Wavelength Assignment Approaches for Wavelength-Routed Optical WDM Networks, Optical Networks Magazine: 47-60 (2000). 